TRANSACTIONS OF THE

AMERICAN MATHEMATICAL SOCIETY

Volume 352, Number 7, Pages 3339-3356

S 0002-9947(00)02454-5

Article electronically published on March 24, 2000

\title{
THE CHARACTERS OF THE GENERALIZED STEINBERG REPRESENTATIONS OF FINITE GENERAL LINEAR GROUPS ON THE REGULAR ELLIPTIC SET
}

\author{
ALLAN J. SILBERGER AND ERNST-WILHELM ZINK
}

\begin{abstract}
Let $k$ be a finite field, $k_{n} \mid k$ the degree $n$ extension of $k$, and $G=\mathrm{GL}_{n}(k)$ the general linear group with entries in $k$. This paper studies the "generalized Steinberg" (GS) representations of $G$ and proves the equivalence of several different characterizations for this class of representations. As our main result we show that the union of the class of cuspidal and GS representations of $G$ is in natural one-one correspondence with the set of Galois orbits of characters of $k_{n}^{\times}$, the regular orbits of course corresponding to the cuspidal representations. Besides using Green's character formulas to define GS representations, we characterize GS representations by associating to them idempotents in certain commuting algebras corresponding to parabolic inductions and by showing that GS representations are the sole components of these induced representations which are "generic" (have Whittaker vectors).
\end{abstract}

Let $k=\mathbb{F}_{q}$ be a finite field of cardinality $q$, let $\bar{k}\left|k_{m}\right| k$ be, respectively, an algebraic closure of $k$ and the degree $m$ extension of $k$ contained in $\bar{k}$. Let $\phi: x \mapsto x^{q}$ denote the Frobenius automorphism of $\bar{k} \mid k$ and of any subextension $k_{m} \mid k$. Let $G=\mathrm{GL}_{n}(k)$ be the group of non-singular $n \times n$ matrices with entries in $k$. Also write $G_{m}=\mathrm{GL}_{m}(k)$ for any $m \geq 1$.

In a signally important paper published in 1955 GR J. A. Green showed how to calculate formulas for the irreducible characters of $G$. In Green's work appeared for the first time general character formulas for the cuspidal representations and for a family of "generalized Steinberg" (GS) representations.

For their study of the "level zero" discrete series characters of unit groups of simple algebras over a $\mathrm{p}$-adic field the authors need diverse characterizations of cuspidal and GS characters of finite general linear groups and to be able to pass between these different characterizations. In this paper we give these characterizations and prove their equivalence.

It is fruitful to view the set of GS representations as a class of representations of $G$ which contains the class of cuspidal representations as a subclass. Of course the cusp form property, the property of not being a component of $\operatorname{Ind}_{U}^{G} \mathbf{1}$ for any unipotent radical $U \neq(I)$ of a parabolic subgroup of $G$, clearly distinguishes the class of cuspidal representations from all other representations of $G$. However, other important properties which are usually associated to the class of cuspidal representations generalize to the union of the two classes of representations of $G$.

Received by the editors May 26, 1997 and, in revised form, April 18, 1998 and June 26, 1998. 1991 Mathematics Subject Classification. Primary 22E50, 11T24.

Key words and phrases. Reductive group, general linear group, finite field, character, unitary representation, Steinberg representation, Whittaker vector, generic representation. 
For instance Green gives a single character formula, with an accompanying degree formula, which applies to both classes of representations, mutatis mutandis. Both the cuspidal and the GS representations are "generic", in the sense that they have "Whittaker vectors" (cf. §5). The main result of this paper (Theorem 6.1) reads out of Green's results the fact that on the regular elliptic set of $G$ the characters of the GS representations, like the cuspidal characters, are represented by familiar character sums. It is important that the character sum which represents the value of the character of a GS representation on the regular elliptic set of $G=G_{n}$ is "norm related" to the character sum which represents the character of the corresponding "cuspidal base representation" (cf. §3) on the regular elliptic set of $G_{v}$ $(v \mid n)$ (see equation (8)). Corollary 6.2 elaborates this relationship in presenting a natural one-one correspondence between the set of cuspidal and generalized Steinberg representations of $G$ and the set of Galois orbits of characters of $k_{n}^{\times}$, the cuspidal characters corresponding to the orbits which consist of regular characters of $k_{n}^{\times}$. The authors use these structural facts to "weakly explicitly match" (except for an unramified twist) the "level zero" discrete series characters of the unit groups between the various $\mathrm{p}$-adic simple algebras of the same reduced degree over the same center ([SZ2]).

To prove the one-one correspondence of Corollary 6.2 the authors need a preliminary result (Theorem 1.1) which implies that the restrictions of the GS characters to the regular elliptic set of $G$ are pairwise distinct functions. The proof of Theorem 1.1 given in this paper depends upon a classical result of Zsigmondy ZS (see also [ART] and [BV]). The authors wish to thank a referee for pointing out these references and the simplified proof of Theorem 1.1 which Zsigmondy's result implies. Our first section also introduces certain character sums which represent the values of GS representations on the regular elliptic set and discusses the connections between these character sums and the parameter set of Macdonald for the set of GS representations of $G$ ([MAC $(1.4, \mathrm{ii})])$. The paper's concluding Remark 6.3, using Macdonald's parameter set, describes a natural parameterization for the set of all cuspidal and GS representations of finite general linear groups.

Most of this paper is devoted to proving that the different ways of characterizing GS representations lead to the same class of representations. In $\S 2$ we recall Green's character formulas for cuspidal representations. In $\S 3$ GS and "generalized trivial" (GT) representations are defined via Green's character formulas; we also prove that the GS and GT representations are the components of certain parabolically induced representations which occur simply (Lemma 3.1). In $\S 4$ we use the Hecke algebra isomorphism given in Howe's lectures [HM, Chapter 1] to associate idempotents in the commuting algebras of the associated parabolically induced representations to the GS and GT components. We use the degrees of these representations and a calculation based on Schur orthogonality to assign these idempotents to the correct representations (Proposition 4.2).

In $\S 5$ we recall classical results concerning generic representations of finite general linear groups, results which have been long known to experts in representation theory, and we use these results to obtain yet another useful characterization of the class of GS representations (Corollary 5.7). Though this characterization is also known by the experts, we shall give a proof here. We begin the section with an old argument, due to either Gelfand and Graev or Gelfand and Kazhdan, which proves that cuspidal representations are generic (Lemma 5.2). Next comes a more precise characterization of cuspidal representations (Lemma 5.3) which was found 
by S. I. Gelfand GE1, GE2. We then prove the $\mathrm{GL}_{n}(k)$ analogue of a result of Rodier (Theorem 5.5) ROD. Using these results, we reprove Gelfand/Graev's theorem that an irreducible representation of $\mathrm{GL}_{n}(k)$ has at most a one-dimensional subspace associated to a given generic character (Corollary 5.6). As the last result of the section, we obtain our characterization of GS representations as the only generic components of certain parabolically induced from cuspidal representations.

The concluding $\S 6$ states and proves our main result, then presents its corollary and the final remark.

Although in this paper we treat only the representation theory of the group $\mathrm{GL}_{n}(k)$ and our work involves only the classical results mentioned above, we should try to place this paper within the context of the important results regarding the representation theory of finite reductive groups which have appeared during the past more than twenty years. In [DL] Deligne and Lusztig constructed families of virtual characters for reductive groups over finite fields by using $\ell$-adic cohomology. Let $\mathcal{G}$ be a connected reductive $k$-group. Let $\mathcal{T}$ be a $\langle\phi\rangle$-stable maximal torus of $\mathcal{G}$ and $\theta$ an irreducible character of the group of fixed points $\mathcal{T}^{\phi}$ of $\mathcal{T}$. To this data Deligne and Lusztig associate a virtual character $R_{\mathcal{T}}^{\mathcal{G}}(\theta)$ of $\mathcal{G}^{\phi}$ which, up to sign, is irreducible if $\theta$ is in general position ([CAR, Theorem 7.3.4], [DL] Theorem 6.8]). In particular, $\pm R_{\mathcal{T}}^{\mathcal{G}}(\theta)$ is an irreducible cuspidal character if $\theta$ is in general position and $\mathcal{T}$ lies in no proper $\langle\phi\rangle$-stable parabolic subgroup of $\mathcal{G}$ (CAR, Theorem 9.3.2], [DL, Theorem 8.3]). This construction produces all the irreducible cuspidal characters of $\mathrm{GL}_{n}(k)$. Let $\mathcal{L}$ be a $\langle\phi\rangle$-stable Levi subgroup of $\mathcal{G}$ and let $\pi$ be a cuspidal representation of $\mathcal{L}^{\phi}$. Let $\mathcal{P}=\mathcal{L} \ltimes \mathcal{U}_{\mathcal{L}}$ be a $\langle\phi\rangle$ stable parabolic subgroup of $\mathcal{G}$ with $\mathcal{L}$ as Levi factor and consider the induced representation $I\left(\mathcal{P}^{\phi}, \pi\right)$. In HL1] (see also [L, Chapter 8]) Howlett and Lehrer have studied the commuting algebra of $I\left(\mathcal{P}^{\phi}, \pi\right)$ and in [HL2 have considered irreducible characters which correspond to the trivial and sign characters of Coxeter groups of the form $N_{\mathcal{G}}(\mathcal{L}, \pi) / \mathcal{L}$. Thus they have considered analogues and generalizations of the "generalized Steinberg" (GS) and "generalized trivial" (GT) representations which are studied here. These representations are interchanged by "Alvis-Curtis duality" (see CUR]). Concerning the theory of "generic representations", discussed in $\S 5$ for the special case of $\mathrm{GL}_{n}\left(\mathbb{F}_{q}\right)$, it should be remarked that [DL, Theorem 10.7] (see also CAR, Proposition 8.4.7]) gives a complete description of the irreducible generic characters of general $\mathcal{G}^{\phi}(\mathcal{G}$ with connected center), expressing them as linear combinations of Deligne-Lusztig characters $R_{\mathcal{T}}^{\mathcal{G}}(\theta)$. In principle, this description can also be used to obtain the results of our $\S 5$. Bernshtein and Zelevinskii [BZ] give a full account of the theory of generic representations for $\mathrm{GL}_{n}$ in the local field case and they remark that similar results hold for $\mathrm{GL}_{n}(k)$.

\section{Galois Orbit Sums of Characters on Finite Fields and Macdonald's Parameter Set}

As already mentioned, the characters of the GS representations are represented on the regular elliptic set of $G$ by familiar character sums. Let $X$ denote the group of characters of $k_{n}^{\times}$. For any $\chi \in X$ we write $[\chi]$ for the $\langle\phi\rangle$-orbit of $\chi$. The character sums alluded to are the sums of the form

$$
S(\chi)=\sum_{\eta \in[\chi]} \eta
$$


Since the set $X$ is a basis for the space of complex-valued functions on $k_{n}^{\times}$, the set of character sums $S(\chi)$, regarded as a set of complex-valued functions on $k_{n}^{\times}$, is a linearly independent set. Let $\mathcal{R}=\mathcal{R}_{n}$ be the set of primitive elements of $k_{n} \mid k$. Identifying the set of regular elliptic conjugacy classes of $\mathrm{GL}_{n}(k)$ with the set of $\langle\phi\rangle$-orbits in $\mathcal{R}$ under any embedding of $k_{n}^{\times}$into $\mathrm{GL}_{n}(k)$, we may represent the values of the generalized Steinberg characters on the regular elliptic set of $\mathrm{GL}_{n}(k)$ as the set of functions of the form $(-1)^{n-1} S(\chi)$. We regard the character sums $S(\chi)$ as functions on $\mathcal{R}$; it is natural then to ask whether this data determines the character orbit $[\chi]$.

\section{Theorem 1.1.}

(i) For any $\chi, \chi^{\prime} \in X$ the restrictions to the set $\mathcal{R} \subset k_{n}^{\times}$of the functions $S(\chi)$ and $S\left(\chi^{\prime}\right)$ are equal if and only if $[\chi]=\left[\chi^{\prime}\right]$.

(ii) There is no $\langle\phi\rangle$-orbit $[\chi] \subset X$ such that the function $S(\chi)$ is identically zero on $\mathcal{R}$.

Example. Consider the quadratic extension $\mathbb{F}_{4} \mid \mathbb{F}_{2}$ with the automorphism $\phi(x)=$ $x^{2}$. The character group $X=X\left(\mathbb{F}_{4}^{\times}\right)$splits into two $\langle\phi\rangle$-orbits $\left[\chi_{0}\right],\left[\chi_{1}\right]$ of lengths 1 and 2 respectively. Restricted to $\mathcal{R}=\mathbb{F}_{4}-\mathbb{F}_{2}$, the orbit sum $S\left(\chi_{0}\right)=\chi_{0}$ has constant value 1 , while $S\left(\chi_{1}\right)=\chi_{1}+\chi_{2}$ has the constant value -1 . Thus the two functions, though not linearly independent as functions on $\mathcal{R}$, are different functions on $\mathcal{R}$ and neither is identically zero.

Proof. If $[\chi]=\left[\chi^{\prime}\right]$, then $S(\chi)=S\left(\chi^{\prime}\right)$ on all of $k_{n}^{\times}$, therefore in particular on $\mathcal{R}$. We have to prove only the converse. Assume that $[\chi] \neq\left[\chi^{\prime}\right]$ but $S(\chi)-S\left(\chi^{\prime}\right)$ vanishes on $\mathcal{R}$. In this case the restriction to $k_{n}^{\times}-\mathcal{R}$ cannot be zero. Let $Y \subset X$ be the group of characters which are identically 1 on $k_{n}^{\times}-\mathcal{R}$. Then for every $\eta \in Y$ we have $\eta\left(S(\chi)-S\left(\chi^{\prime}\right)\right)=S(\chi)-S\left(\chi^{\prime}\right)$ as functions on $k_{n}^{\times}$. Since the set $X$ is a basis of the space of complex-valued functions on $k^{\times}$, it follows that each of the orbits $[\chi]$ and $\left[\chi^{\prime}\right]$ is stable under multiplication by elements of $Y$. This implies that $|Y| \leq|[\chi]|$, where $|Y|$ denotes the order of the group $Y$. Since any orbit of the cyclic group $\langle\phi\rangle /\left\langle\phi^{n}\right\rangle$ acting in $X=X\left(k_{n}^{\times}\right)$must have length dividing $n$, from cancellation in the group $X$ we infer that $|Y| \leq n$. Thus we shall have a contradiction as soon as we prove that $|Y|>n$. From the hypothesis $S(\chi)=0$ on $\mathcal{R}$, the same argument also proves Theorem 1.1(ii).

Lemma 1.2. Assume that $n>1$ and let $\Phi_{n}(x)=\prod_{d \mid n}\left(x^{n / d}-1\right)^{\mu(d)}$ be the $n$ th cyclotomic polynomial. Then $|Y|$ divides $\Phi_{n}(q)$ for all $n$ and $q$. Moreover, $|Y| \geq \Phi_{n}(q)>n$ for all $(n, q) \neq(6,2)$.

Remarks. 1. Since $\Phi_{6}(x)=x^{2}-x+1$, we have $\Phi_{6}(2)=3 \leq 6 ; \Phi_{n}(q)>n$ definitely fails in the case $(n, q)=(6,2)$. Thus we have to verify separately that Theorem 1.1 is true when $(n, q)=(6,2)$. We delay this for the moment.

2. A referee pointed out that for Theorem 1.1 it is enough to know that $|Y| \geq$ $\Phi_{n}(q)$. It is easier to prove this than $|Y|=\Phi_{n}(q)$, which we showed in our original proof of Theorem 1.1. We also gave a longer proof that $\Phi_{n}(q)>n$ for $(n, q) \neq$ $(6,2)$. The referee pointed out that this assertion follows from results of Zsigmondy [ZS] (see also [ART, $\S 1$, Corollary 2] and [BV]). Zsigmondy proved that for any $(n, q) \neq(6,2)$ or $(2,3)(n>1)$ there is always a prime $\ell$ such that $\ell \mid \Phi_{n}(q)$ and $\ell \nmid \Phi_{i}(q)(\forall i<n)$, i.e. such that $\ell \mid q^{n}-1$ and $\ell \nmid q^{i}-1(\forall i<n)$. We reproduce the referee's proof of Lemma 1.2. 
Proof. Since $x^{m}-1=\prod_{d \mid m} \Phi_{d}(x)$ for any $m \geq 1$, it follows that $q^{m}-1$ divides the product $\prod_{d \mid n, d<n} \Phi_{d}(q)$ for all $m \mid n, m<n$. Thus $\operatorname{lcm}_{m \mid n, m<n}\left\{q^{m}-1\right\}$ also divides $\prod_{d \mid n, d<n} \Phi_{d}(q)$. On the other hand, $|Y|=\left(q^{n}-1\right) / \mathrm{lcm}_{m \mid n, m<n}\left\{q^{m}-1\right\}$ and $\Phi_{n}(q)=\left(q^{n}-1\right) / \prod_{d \mid n, d<n} \Phi_{d}(q)$. Therefore, $\Phi_{n}(q)$ divides $|Y|$ too, so $|Y| \geq \Phi_{n}(q)$.

We next show that $\Phi_{n}(q)>n$ follows from the fact that there exists a prime $\ell$ such that $\ell \mid q^{n}-1$ and $\ell \nmid q^{i}-1(\forall i<n)$ ([ZS], [BV], [ART]). Clearly, the existence of $\ell$ implies that $\ell \mid \Phi_{n}(q)$, so $\Phi_{n}(q) \geq \ell$. "Fermat's Little Theorem", together with $(\ell, q)=1$, implies that $\ell \mid q^{\ell-1}-1$. Thus from Zsigmondy, Birkhoff/Vandiver, and Artin we have $\ell>n$. Since $\Phi_{n}(q) \geq \ell>n, \Phi_{n}(q)>n$. To complete the proof of Lemma 1.2 we note that $\Phi_{2}(3)=3+1>2$.

It follows from Lemma 1.2 and the above argument that we have to verify Theorem 1.1 only in the case $(n, q)=(6,2)$. Write $K=\mathbb{F}\left(2^{6}\right)$ and $k=\mathbb{F}(2)$. Fix a generator $\chi_{1}$ of the cyclic group $X=X\left(K^{\times}\right)$; thus $a \in(\mathbb{Z} / 63 \mathbb{Z})^{+} \mapsto \chi_{1}^{a} \in X$ defines an isomorphism. In the present instance $Y$ consists of the three characters which correspond to the multiples of 21 in $(\mathbb{Z} / 63 \mathbb{Z})^{+}$. The action of the Frobenius on $X$ is given by $\chi \mapsto \chi^{2}$, i.e. $a \mapsto 2 a$ in $(\mathbb{Z} / 63 \mathbb{Z})^{+}$.

If $S(\chi)=S\left(\chi^{\prime}\right)$ on $\mathcal{R}$ or $S(\chi)=0$ on $\mathcal{R}$, then $\eta[\chi]=[\chi]$ for all $\eta \in Y$. Hence $[\chi] \subset X$ corresponds to an orbit $[a]=\left\{a, 2 a, \ldots, 2^{5} a\right\} \subset \mathbb{Z} / 63 \mathbb{Z}$ such that $[a]+21=[a]$. It is easy to see that the only orbit satisfying this condition is the orbit $[a]=[7]$, which is of length 6 . This implies Theorem 1.1(i), since there is only one $Y$-invariant character sum $S(\chi)$, namely the sum corresponding to $\chi:=\chi_{1}^{7}$. To verify Theorem 1.1(ii) we have to prove $S(\chi) \neq 0$ for some $x \in \mathcal{R} \subset K^{\times}$. Since $\eta S(\chi)=S(\chi)$ for $\eta \in Y$, we see that $S(\chi)(x) \neq 0$ implies that $\eta(x)=1$, i.e. $x \in\left(K^{\times}\right)^{3}$. Let $\theta:=\chi(x)=\chi_{1}^{7}\left(y^{3}\right)=\chi_{1}\left(y^{21}\right)$. Then $\theta^{3}=1$, and $\theta=1$ if and only if $y^{21}=x^{7}=1$. In the last case, $S(\chi)(x)=|[\chi]|=6$, whereas for $x^{7} \neq 1$ we obtain $\theta+\theta^{2}=-1$, hence $S(\chi)(x)=-3$. Thus the support of $S(\chi)$ is precisely $\left(K^{\times}\right)^{3}$, and there are regular elements in $\left(K^{\times}\right)^{3}$ because $\mathbb{F}\left(2^{3}\right)^{\times} \cup \mathbb{F}\left(2^{2}\right)^{\times}$contains fewer than twenty-one elements.

We close this section by reformulating Theorem 1.1 for the limit of the groups $X_{m}:=X\left(k_{m}^{\times}\right)(m \geq 1)$. Fix an algebraic closure $\bar{k} \mid k$. The Frobenius $\phi:\left\{x \mapsto x^{q}\right\}$ acts on $\bar{k}$ and the fixed field $k_{m}$ of $\left\langle\phi^{m}\right\rangle$ is the extension of degree $m$ of $k$.

For $m \mid m^{\prime}$ we have the surjective norm mapping $N_{m \mid m^{\prime}}: k_{m^{\prime}}^{\times} \rightarrow k_{m}^{\times}$. We let $\tilde{k}:=\lim _{k_{m}}^{\times}$be the projective limit with respect to the norm maps. For $\tilde{x} \in \tilde{k}$ we write $\tilde{x}=\left(x_{m}\right)$, where $x_{m}$ is the projection of $\tilde{x}$ on $k_{m}^{\times}$. By Zorn's Lemma the surjectivity of the norm maps implies the surjectivity of the projection maps.

Dually let $X_{m}=X\left(k_{m}^{\times}\right)$and $N_{m \mid m^{\prime}}^{*}: X_{m} \rightarrow X_{m^{\prime}}$. The maps $N_{m \mid m^{\prime}}^{*}$ being injective, we may consider the direct limit $\tilde{X}=\underline{\varliminf} X_{m}$ as a quotient of the disjoint union $X=\bigsqcup_{m=1}^{\infty} X_{m}$, namely $\tilde{X}=X / \sim$, where $\eta_{m_{1}} \sim \eta_{m_{2}}$ if $N_{m_{1} \mid m_{1} m_{2}}^{*}\left(\eta_{m_{1}}\right)=$ $N_{m_{2} \mid m_{1} m_{2}}^{*}\left(\eta_{m_{2}}\right)$.

Sometimes the elements $\tilde{\eta} \in \tilde{X}$ are considered as subsets of $X$. Note that $\tilde{\eta} \cap X_{m}$ is either void or a single element $\eta_{m}$. Canonically, we have the character group $\tilde{X}=X(\tilde{k})$, where $\tilde{\eta}(\tilde{x})=\eta_{m}\left(x_{m}\right)$ if $\eta_{m} \in \tilde{\eta}$. The Frobenius acts on $k_{m}^{\times}$and $X_{m}$ such that ${ }^{\phi} \eta_{m}\left({ }^{\phi} x_{m}\right)=\eta_{m}\left(x_{m}\right)$. This induces a natural $\langle\phi\rangle$-action on $\tilde{k}$ and $\tilde{X}$. We remark that the action of $\langle\phi\rangle$ on $\tilde{X}$ has orbits $[\tilde{\eta}]$ of finite length, namely $|[\tilde{\eta}]|=\left|\left[\eta_{m}\right]\right|$ if $\eta_{m} \in \tilde{\eta}$. If $|[\tilde{\eta}]|=s$, then $X_{n} \cap \tilde{\eta} \neq \emptyset$ if and only if $s \mid n$, and $X_{s} \cap \tilde{\eta}$ is the unique regular element of $\tilde{\eta}$. 
Let $\tilde{\chi} \in \tilde{X}$. Define

$$
S(\tilde{\chi})=S([\tilde{\chi}])=\sum_{\tilde{\eta} \in[\tilde{\chi}]} \tilde{\eta}
$$

and consider this as a function on $\tilde{k}$ such that

$$
S(\tilde{\chi})(\tilde{x})=S\left(\chi_{m}\right)\left(x_{m}\right)=\sum_{\eta_{m} \in\left[\chi_{m}\right]} \eta_{m}\left(x_{m}\right) \quad\left(\chi_{m} \in \tilde{\chi}\right) .
$$

Since the projection map $\tilde{x} \in \tilde{k} \mapsto x_{m} \in k_{m}^{\times}$is surjective, the function $S\left(\chi_{m}\right)$ is recovered from $S(\tilde{\chi})$. We write $S(\tilde{\chi})_{m}:=S\left(\chi_{m}\right)$ if $|[\tilde{\chi}]| \mid m$. Let $\mathcal{R}_{m}$ denote the set of primitive elements of $k_{m} \mid k$. Then:

Corollary 1.3. Let $\tilde{\chi}, \tilde{\chi}^{\prime} \in \tilde{X}$ with $\langle\phi\rangle$-orbits of length $s, s^{\prime}$, respectively. The following are equivalent:

(i) $[\tilde{\chi}]=\left[\tilde{\chi}^{\prime}\right]$.

(ii) $S(\tilde{\chi})=S\left(\tilde{\chi}^{\prime}\right)$ as $\mathbb{C}$-valued functions on $\tilde{k}$.

(iii) For all common multiples $m$ of $s, s^{\prime}$ the mappings $S(\tilde{\chi})_{m}$ and $S\left(\tilde{\chi}^{\prime}\right)_{m}$ of $\mathcal{R}_{m} \rightarrow$ $\mathbb{C}$ are equal.

(iv) For some common multiple $m$ of $s, s^{\prime}$ the mappings $S(\tilde{\chi})_{m}$ and $S\left(\tilde{\chi}^{\prime}\right)_{m}$ of $\mathcal{R}_{m} \rightarrow \mathbb{C}$ are equal.

Proof. The implications (i) $\rightarrow$ (ii) $\rightarrow$ (iii) $\rightarrow$ (iv) are obvious. Concerning (iv) $\rightarrow$ (i) we remark that $S(\tilde{\chi})_{m}=S\left(\tilde{\chi}^{\prime}\right)_{m}$ on $\mathcal{R}_{m}$ means $S\left(\chi_{m}\right)=S\left(\chi_{m}^{\prime}\right)$ on $\mathcal{R}_{m}$, where $\chi_{m} \in \tilde{\chi}$ and $\chi_{m}^{\prime} \in \tilde{\chi}^{\prime}$. Hence, $\left[\chi_{m}\right]=\left[\chi_{m}^{\prime}\right]$ by Theorem 1.1; we conclude that $[\tilde{\chi}]=\left[\tilde{\chi}^{\prime}\right]$.

\section{Cuspidal Characters}

To be able to read the remainder of this paper the reader will have to keep Green's paper [GR] nearby in order to be able to follow our references to it.

Let $\tilde{\chi}_{d} \in \tilde{X}$ and assume that $\left|\left[\tilde{\chi}_{d}\right]\right|=d \geq 1$. We assume, as we may, that $\chi_{d} \in X_{d} \cap \tilde{\chi}_{d}$ and that $\chi_{d}$ is the only regular character in $\tilde{\chi}_{d}$. The orbit $\left[\chi_{d}\right] \subset X_{d}$ consists of regular characters.

Using Green's work, we associate to the $\langle\phi\rangle$-orbit $\left[\chi_{d}\right]$ a cuspidal character of $G_{d}$, specifically the character $U=(-1)^{d-1} I_{d}^{k}[1]=(-1)^{d-1} J_{d}(k)$ given by GR. pp. 430-431, Example (ii); cf. also pp. 417 (top) and 433] (In Green's notation $\theta^{k}=\chi_{d}$ GR, p. 431 or 433$\left.].\right)$. The factor $(-1)^{d-1}$ is needed to convert a virtual character into a true irreducible character (GR, p. 437 mid-page; see p. 409, Definition 2.2 for the function $\left.\left.\phi_{d-1}\right]\right)$. The degree of any cuspidal representation of $G_{d}(d>1)$ is $\prod_{1 \leq j<d}\left(q^{j}-1\right)$ ([GR, p. 431 ; see p. 420 for $\left.\left.k(\lambda, q)\right]\right)$. Green proves that this character (or family of characters) is cuspidal in the sense that this character occurs as a component of no character constructed in the standard way by parabolic induction. He also proves that all (irreducible) cuspidal characters are of the form $(-1)^{d-1} I_{d}^{k}[1]$ for some choice of $k$.

Green's results [GR, p. 431] imply the existence of a one-one correspondence between cuspidal characters of $G_{d}$ and orbits $\left[\eta_{d}\right]$ of regular characters of $k_{d}^{\times}$such that the cuspidal character $U=U\left(\left[\eta_{d}\right]\right)$ satisfies

$$
U(\gamma)=U_{\eta_{d}}(\gamma)=(-1)^{d-1} S\left(\eta_{d}\right)(\gamma)
$$

for all regular elliptic elements $\gamma \in G_{d}$. We identify regular elliptic elements of $G_{d}$ with primitive elements of the field extension $k_{d} \mid k$. The function $S\left(\chi_{d}\right): \mathcal{R}_{d} \rightarrow \mathbb{C}$ 
with domain the set $\mathcal{R}_{d}$ of primitive elements of $k_{d} \mid k$ is defined from the character sum of (1).

\section{Generalized Steinberg and Generalized Trivial Characters}

To relate the standard construction of GS representations to Green's character formula let $\chi_{s} \in X_{s}(1 \leq s<n, s \mid n)$ be a regular character and let $\sigma=\sigma_{\chi_{s}}$ be the cuspidal representation of $G_{s}$ with the character $U_{\chi_{s}}$. To be consistent with Green's notation we assume that the character $U_{\chi_{s}}$ of $G_{s}$ is $(-1)^{s-1} I_{s}^{k}[1]$, the values of which on the regular elliptic set of $G_{s}$ are given by $(-1)^{s-1} S\left(\chi_{s}\right)$ (cf. (1)) with $\chi_{s}=\theta^{k}$. As before, the factor $(-1)^{s-1}$ converts a virtual character into a true character.

Regard $\left[G_{s}\right]^{v}=L(s v=n)$ as a block diagonal subgroup of $G$ and let $P=$ $L \ltimes U$ be the parabolic subgroup with $L$ as a Levi factor which contains the upper triangular subgroup of $G$. Regard the $v$-fold tensor power $\sigma^{\otimes v}$ as a representation of $L$ inflated to a representation of $P$. Let $I\left(P, \sigma^{\otimes v}\right)=\operatorname{Ind}_{P}^{G} \sigma^{\otimes v}$.

We call $\sigma$ the cuspidal base representation which corresponds to the components of $I\left(P, \sigma^{\otimes v}\right)$. Since the representations parabolically induced from different cuspidal representations or non-conjugate Levi factors of $G$ are disjoint, the cuspidal base representation is an invariant which distinguishes the components of $I\left(P, \sigma^{\otimes v}\right)$ from other irreducible representations of $G$.

In Green's terminology, the character of $I\left(P, \sigma^{\otimes v}\right)$ is a "basic character" of the form $\left.(-1)^{n-v} B^{s \cdot\left\{1^{v}\right.}\right\}(k)$ GR, p. 433, Definition 6.2]. The factor $(-1)^{n-v}=$ $(-1)^{(s-1) v}$ results from our earlier conversion of the virtual cuspidal character $I_{s}^{k}[1]$ into a true character, then tensoring it $v$ times. The argument " $\mathrm{k}$ " means the vector $(k, k, \cdots, k)$ of length $v$.

It is known that the commuting algebra of $I\left(P, \sigma^{\otimes v}\right)$ is isomorphic to the group algebra $\mathbb{C}\left(\mathfrak{S}_{v}\right)$, where $\mathfrak{S}_{v}$ denotes the symmetric group on $v$ letters (cf. IW. p. 81] and [HM, p. 11, Theorem 5.1]). This means that the inequivalent component representations of $I\left(P, \sigma^{\otimes v}\right)$ correspond bijectively to the characters of $\mathfrak{S}_{v}$. This correspondence is made explicit in Green's character formulas, where expressions of the form $\chi_{\pi}^{\lambda}$ denote the values of characters of $\mathfrak{S}_{v}$. In these formulas $\lambda$ and $\pi$ both denote partitions of $v, \lambda$ used to parameterize characters and $\pi$ to parameterize conjugacy classes of $\mathfrak{S}_{v}$. Since the degrees of the characters of $\mathfrak{S}_{v}$ equal the multiplicities of the components of $I\left(P, \sigma^{\otimes v}\right)$, there are two irreducible components of $I\left(P, \sigma^{\otimes v}\right)$ which occur simply, one corresponding to the trivial character and the other to the sign character of $\mathfrak{S}_{v}$. These characters or representations we shall refer to as the "generalized trivial" (GT) and "generalized Steinberg" (GS) components of $I\left(P, \sigma^{\otimes v}\right)$.

We now recall these characters as Green defines them in GR, p. 437, Definition 7.3] 1 These two characters are also specifically referred to following [GR, p. 438, Lemma 7.4]. In terms of "basic characters" the "generalized trivial" (GT) character is

$$
(-1)^{n-v} I_{s}^{k}[v]=(-1)^{n-v} \sum_{|\pi|=v} \frac{1}{z_{\pi}} \chi_{\pi}^{v} B^{s \cdot \pi}\left(k \frac{\pi}{v}\right)
$$

\footnotetext{
${ }^{1}$ In this definition Green gives a character for each partition of $v$ or character of $\mathfrak{S}_{v}$. Green is certainly not guilty of using the names "generalized trivial" and "generalized Steinberg", although he singles these two characters out and discusses the connection with Steinberg's work in [GR] p. $438]$.
} 
with the degree

$$
\frac{\prod_{j=1}^{n}\left(q^{j}-1\right)}{\prod_{j=1}^{v}\left(q^{s j}-1\right)}
$$

and the "generalized Steinberg" (GS) character is

$$
(-1)^{n-v} I_{s}^{k}\left[1^{v}\right]=(-1)^{n-v} \sum_{|\pi|=v} \frac{1}{z_{\pi}} \chi_{\pi}^{1^{v}} B^{s \cdot \pi}\left(k \frac{\pi}{v}\right)
$$

with the degree 2

$$
q^{n(v-1) / 2} \frac{\prod_{j=1}^{n}\left(q^{j}-1\right)}{\prod_{j=1}^{v}\left(q^{s j}-1\right)}
$$

In (2) and (4) the sums extend over partitions $\pi$ of $v, z_{\pi}=1^{p_{1}} p_{1} ! 2^{p_{2}} p_{2} ! \cdots$ when $\pi=1^{p_{1}} 2^{p_{2}} \ldots$ is a partition with $p_{1}$ parts $1, p_{2}$ parts 2 , etc., $\chi_{\pi}^{v}=1$ (GR, p. 423 , Definition 4.11]), and $\chi_{\pi}^{1^{v}}$ is the sign character of the symmetric group $\mathfrak{S}_{v}$ evaluated at the conjugacy class corresponding to $\pi$. In the sense that the values of $\chi_{\pi}^{v}$ (the trivial character) and $\chi_{\pi}^{1^{v}}$ (the sign character) of $\mathfrak{S}_{v}$ appear in the formulas for the GT and GS characters, the two characters correspond, respectively, to the trivial and sign characters of $\mathfrak{S}_{v}$.

The purpose of Lemma 3.1 is to show that the two components of $I\left(P, \sigma^{\otimes v}\right)$ which occur simply are indeed the representations with the respective characters $(-1)^{n-v} I_{s}^{k}[v]$ and $(-1)^{n-v} I_{s}^{k}\left[1^{v}\right]$.

Lemma 3.1. With respect to the measure on $G$ which assigns $G$ measure one the inner products

$$
\left((-1)^{n-v} I_{s}^{k}[v],(-1)^{n-v} B^{s \cdot\left\{1^{v}\right\}}(k)\right)=\left((-1)^{n-v} I_{s}^{k}\left[1^{v}\right],(-1)^{n-v} B^{s \cdot\left\{1^{v}\right\}}(k)\right)=1 .
$$

Proof. By GR, p. 435, Lemma 6.3] the inner product

$$
\left(B^{s \cdot\left\{1^{v}\right\}}(k), B^{s \cdot \pi}\left(k \frac{\pi}{s}\right)\right)=0
$$

for $\pi \neq 1^{v}$, and

$$
\left((-1)^{n-v} B^{s \cdot\left\{1^{v}\right\}}(k),(-1)^{n-v} B^{s \cdot\left\{1^{v}\right\}}(k)\right)=v !,
$$

since $v$ ! is the dimension of the commuting algebra of $I\left(P, \sigma^{\otimes v}\right)$. Therefore,

$$
\begin{gathered}
\left((-1)^{n-v} I_{s}^{k}\left[1^{v}\right],(-1)^{n-v} B^{s \cdot\left\{1^{v}\right\}}(k)\right) \\
=\left((-1)^{n-v} \sum_{|\pi|=v} \frac{1}{z_{\pi}} \chi_{\pi}^{1^{v}} B^{s \cdot \pi}\left(k \frac{\pi}{s}\right),(-1)^{n-v} B^{s \cdot\left\{1^{v}\right\}}(k)\right)=1,
\end{gathered}
$$

since $z_{1^{v}}=v$ ! and $\chi_{1^{v}}^{1^{v}}=1$. Similarly,

$$
\left((-1)^{n-v} I_{s}^{k}[v],(-1)^{n-v} B^{s \cdot\left\{1^{v}\right\}}(k)\right)=1 .
$$

\footnotetext{
${ }^{2}$ The formula for the dimension of the virtual character $I_{s}^{k}\left[1^{v}\right]$ in the middle of [GR, p. 438] should $\operatorname{read} q^{s C_{v, 2}} \phi_{s v}(q) / \phi_{v}\left(q^{s}\right)$.
} 


\section{GT and GS Representations as Hecke Algebra Characters}

We wish to have another characterization of GT and GS representations. Let $T_{0}$ denote the diagonal subgroup of $G, Z=k^{\times}$the scalar subgroup of $T_{0}$, and $U_{0}$ the upper triangle unipotent subgroup of $G$, consisting of all upper triangular matrices with each diagonal entry equal 1 . Also let $P_{0}=T_{0} \ltimes U_{0}$ denote the upper triangle subgroup of $G$. The commuting algebra of $\operatorname{Ind}_{P_{0}}^{G} \mathbf{1}$ is canonically the convolution algebra $\mathcal{H}_{0}=\mathcal{H}\left(G, P_{0}, \mathbf{1}\right)$ of all left and right $P_{0}$-invariant complexvalued functions on $G$. The algebra $\mathcal{H}_{0}$ has a basis $\left\{h_{w}\right\}_{w \in W_{0}}$, where each of the functions $h_{w}$ is the characteristic function of a double coset $P_{0} w P_{0}, W_{0}$ being the group of permutation matrices $\mathfrak{S}_{n}$. We normalize the invariant measure on $G$ such that $P_{0}$ has unit volume. Then the identity element of $\mathcal{H}_{0}$ is the characteristic function $h_{I}$ of $P_{0}$. If $r=r_{i} \in W_{0}$ is a "reflection with respect to a simple root", i.e. $r=r_{i}$ is the transposition matrix which permutes the $i$-th and $(i+1)$-th components of a standard vector for some $1 \leq i<n$, then

$$
h_{r} * h_{r}=q h_{I}+(q-1) h_{r}
$$

and

$$
h_{r} * h_{w}=h_{r w}
$$

for all $w \in W_{0}$ such that $\ell(r w)=\ell(w)+1$ (cf. [IM], [HM]; " $\ell(w)$ " denotes the "length" of $\left.w \in W_{0}\right)$. Since $\mathcal{H}_{0}$ is isomorphic to the group algebra $\mathbb{C}\left[\mathfrak{S}_{n}\right]$ ([IW]), $\mathcal{H}_{0}$ has exactly two scalar characters. To define the idempotent functions in $\mathcal{H}_{0}$ which give these homomorphisms by convolution let

$$
P(x)=(1+x)\left(1+x+x^{2}\right) \cdots\left(1+x+\cdots x^{n-1}\right)
$$

be the Poincaré polynomial associated to a root system of type $A_{n-1}$. Set

$$
e_{t r}=P(q)^{-1} \sum_{w \in W_{0}} h_{w}
$$

and

$$
e_{s t}=P\left(q^{-1}\right)^{-1} \sum_{w \in W_{0}}\left(-q^{-1}\right)^{\ell(w)} h_{w}
$$

\section{Lemma 4.1.}

$$
h_{w} * e_{t r}=e_{t r} * h_{w}=q^{\ell(w)} e_{t r}
$$

and

$$
h_{w} * e_{s t}=e_{s t} * h_{w}=(-1)^{\ell(w)} e_{s t}
$$

for any $w \in W_{0} ; \ell(w)$ denotes the "length" of $w \in W_{0}$. The functions $e_{t r}$ and $e_{s t}$ are idempotents in $\mathcal{H}_{0}$ which span two-sided, one-dimensional ideals in $\mathcal{H}_{0}$.

Proof. We verify only the assertions which concern $e_{t r}$, as the assertions concerning $e_{s t}$ follow by similar arguments. By formula (7) and the associativity of the multiplication in $\mathcal{H}_{0}$, to verify that $h_{w} * e_{t r}=e_{t r} * h_{w}=q^{\ell(w)} e_{t r}$ it suffices to verify that $h_{r} * e_{t r}=e_{t r} * h_{r}=q e_{t r}$ for $r \in W_{0}$ a reflection corresponding to a simple root. It is also not necessary to verify that $h_{r}$ commutes with $e_{t r}$, since this will be obvious from the argument showing that $h_{r} * e_{t r}=q e_{t r}$. To prove that $h_{r} * e_{t r}=q e_{t r}$ we 
let $S_{0}$ be the subset of $W_{0}$ consisting of all $w \in W_{0}$ such that $\ell(r w)=1+\ell(w)$. Then $e_{t r}=\sum_{w \in S_{0}}\left(h_{w}+h_{r w}\right)$. By (6) and (7),

$$
\begin{aligned}
h_{r} *\left(h_{w}+h_{r w}\right) & =h_{r w}+\left(h_{r} * h_{r}\right) * h_{w} \\
& =h_{r w}+q h_{I} * h_{w}+(q-1) h_{r} * h_{w}=q\left(h_{w}+h_{r w}\right) .
\end{aligned}
$$

This implies that $h_{r} * e_{t r}=q e_{t r}$. To complete the proof that $e_{t r} * e_{t r}=e_{t r}$ it is enough to recall that, in the Poincaré polynomial $P(x)=\sum_{\ell} a_{\ell} x^{\ell}$, the coefficient $a_{\ell}$ is the number of elements of $W_{0}$ with length $\ell$. From the relations $h_{w} * e_{t r}=q^{\ell(w)} e_{t r}$ and $h_{w} * e_{s t}=(-1)^{\ell(w)} e_{s t}$ it follows that the mappings $h \mapsto \lambda_{t r}(h)$ and $h \mapsto \lambda_{s t}(h)$, where $h * e_{t r}=e_{t r} * h=\lambda_{t r}(h) e_{t r}$ and $h * e_{s t}=e_{s t} * h=\lambda_{s t}(h) e_{s t}$ for $h \in \mathcal{H}_{0}$, give homomorphisms of $\mathcal{H}_{0}$ to $\mathbb{C}$. Thus, each of the central idempotents $e_{t r}$ and $e_{s t}$ in the semi-simple algebra $\mathcal{H}_{0}$ spans and projects out a one-dimensional, two-sided ideal of $\mathcal{H}_{0}$.

The function $e_{t r}$ is a constant function on $G$ and is therefore a constant multiple of a matrix coefficient of the "trivial" representation of $G$. Therefore, the idempotent $e_{s t}$ is a multiple of the unique $P_{0}$-bi-invariant matrix coefficient of the Steinberg representation of $G$, since the trivial and the Steinberg representations are the components of $\operatorname{Ind}_{P_{0}}^{G} \mathbf{1}$ which occur simply.

Let $P=L \ltimes U$ be the parabolic subgroup of $G$ already considered above and let $P_{0}$ now denote the upper triangular subgroup of $\mathrm{GL}_{v}\left(k_{s}\right)$. By Howe's theory [HM, loc. cit.] the Hecke algebras $\mathcal{H}=\mathcal{H}\left(G, P, \sigma^{\otimes v}\right)$ and $\mathcal{H}_{0}=\mathcal{H}\left(\mathrm{GL}_{v}\left(k_{s}\right), P_{0}, \mathbf{1}\right)$ are isomorphic. The Howe isomorphisms between these algebras are unique up to automorphisms of the Dynkin diagram of $A_{v-1}$; a Howe isomorphism sends operators corresponding to simple reflections to one another. The algebra $\mathcal{H}$ also has exactly two scalar characters. These characters are also represented by idempotent elements of $\mathcal{H}$. Under a Howe isomorphism the idempotent functions $e_{t r}, e_{s t} \in \mathcal{H}_{0}$ correspond to idempotent functions in $\mathcal{H}$ which do not depend upon the choice of Howe isomorphism. We denote these elements of $\mathcal{H}$ also as $e_{t r}$ and $e_{s t}$, respectively. We want to show that the representations associated to $e_{t r}, e_{s t} \in \mathcal{H}$ are, in the proper order, the GT and GS components of $I\left(P, \sigma^{\otimes v}\right)$.

Proposition 4.2. The $G T$ and $G S$ components of $I\left(P, \sigma^{\otimes v}\right)$ are the components which correspond to the idempotents $e_{t r}$ and $e_{s t}$, respectively.

Proof. Since the GT and GS components are the only components of $I\left(P, \sigma^{\otimes v}\right)$ which occur simply, the submatrices of these representations which transform left and right under $P$ as $\sigma^{\otimes v}$ are, up to scalar multiples, the only functions in $\mathcal{H}$ which give scalar characters of $\mathcal{H}$. Thus, the functions $e_{t r}, e_{s t} \in \mathcal{H}$ are multiples of these submatrices of GT and GS in some order. It is sufficient to compute the degrees of the representations corresponding to $e_{t r}$ and $e_{s t}$ in order to show that they correspond to GT and GS in the right order.

Since the algebra $\mathcal{H}$ is canonically the commuting algebra of the induced representation $I\left(P, \sigma^{\otimes v}\right)$, it has two one-dimensional characters. By the Howe isomorphism theorem the idempotent $e_{t r} \in \mathcal{H}$ is

$$
e_{t r}=P\left(q^{s}\right)^{-1} \sum_{w \in W} h_{w}
$$


and

$$
e_{s t}=P\left(q^{-s}\right)^{-1} \sum_{w \in W}\left(-q^{-s}\right)^{\ell(w)} h_{w}
$$

where

$$
P(x)=(1+x)\left(1+x+x^{2}\right) \cdots\left(1+x+\cdots+x^{v-1}\right)
$$

is the Poincaré polynomial corresponding to a root system of type $A_{v-1}, W \cong \mathfrak{S}_{v}$ may be taken to be the group of permutation matrices $R \otimes I_{s}$, where $R$ is a $v \times v$ permutation matrix and $I_{s}$ denotes the $s \times s$ identity matrix. The functions $h_{w}$ are basis elements for the Hecke algebra which have support on double cosets $P w P$ and correspond to the characteristic functions of $P_{0}$ double cosets under the Howe isomorphism. The Howe isomorphism, which transforms the structure constants as in (6) to $\mathcal{H}$, also depends upon normalizing the Haar measure on $G$ to give $P=L \ltimes U$ unit measure. This also implies that the identity element $h_{I}$ of $\mathcal{H}$ has the value $I_{\sigma \otimes v}$ at the identity $I \in G$. We write $I_{\sigma \otimes v}$ for the identity operator in the representation space of $\sigma^{\otimes v}$.

It is an easy consequence of Schur orthogonality (see, for instance, SZ1, p. 11, Corollary 3.3]) that the dimensions of the representations with matrix-valued idempotents $e_{t r}$ and $e_{s t}$ may be computed by computing the traces of these matrixvalued idempotents at $I \in G$ and multiplying these traces by the index $[G: P]$. Since

$$
h_{I}(I)=I_{\sigma \otimes v},
$$

the dimension of the representation corresponding to $e_{t r}$ is

$$
\begin{gathered}
\operatorname{trace}\left(e_{t r}(I)\right)[G: P]=\frac{\operatorname{dim}\left(\sigma^{\otimes v}\right)}{P\left(q^{s}\right)} \frac{\left|\mathrm{GL}_{n}(k)\right|}{\left|\mathrm{GL}_{s}(k)\right|^{v}|U|} \\
=\frac{\prod_{j=1}^{s-1}\left(q^{j}-1\right)^{v}}{\prod_{j=1}^{v-1}\left(\sum_{i=0}^{j} q^{s i}\right)} \frac{\prod_{j=0}^{n-1}\left(q^{n}-q^{j}\right)}{\prod_{j=0}^{s-1}\left(q^{s}-q^{j}\right)^{v} q^{n(n-s) / 2}} \\
=\frac{\prod_{j=1}^{n}\left(q^{j}-1\right)}{\prod_{j=1}^{v}\left(q^{s j}-1\right)},
\end{gathered}
$$

which is the dimension of the GT representation (cf. (3)). Since $P\left(q^{-s}\right)$ appears in the denominator of $e_{s t}$ instead of $P\left(q^{s}\right)$ and since the degree of the polynomial $P(x)$ is $v(v-1) / 2$, the dimension of the representation corresponding to $e_{s t}$ is $q^{s v(v-1) / 2}=$ $q^{n(v-1) / 2}$ times the dimension of the generalized trivial representation, i.e. the dimension of the GS representation (cf. (5)). Since these dimensions equal the respective dimensions of the irreducible characters $(-1)^{n-v} I_{s}^{k}[v]$ and $(-1)^{n-v} I_{s}^{k}\left[1^{v}\right]$ ([GR, p. 438, Lemma 7.4]), $e_{t r}$ is a matrix block of the representation corresponding to the character $(-1)^{n-v} I_{s}^{k}[v]$ and $e_{s t}$ corresponds to $(-1)^{n-v} I_{s}^{k}\left[1^{v}\right]$.

\section{The GS Representation as the Generic Component of $I\left(P, \sigma^{\otimes v}\right)$}

In this section we review the theory of "Whittaker vectors" for $G$ with the goal of showing that the GS representation is the unique component of $I\left(P, \sigma^{\otimes v}\right)$ which has a Whittaker vector. In principle the results of this section follow from the work of Deligne and Lusztig which has already been sketched and referred to in our Introduction. As already mentioned, the results of this section are for the most 
part classical and all are known to experts. We shall indicate original sources for these results to the extent that we know them. In particular, we shall reprove and use results of Gelfand/Graev GG], Gelfand/Kazhdan GK], S. I. Gelfand [GE1, GE2, and Rodier [ROD]. Bernshtein and Zelevinskii [BZ] prove similar results in the local field case.

A one-dimensional character of $U_{0}$ is called generic if its restriction to every super-diagonal one-parameter subgroup $I+x_{i, i+1}(1 \leq i<n)$ is non-trivial. A representation of $G$ is said to be "generic" or to have a "Whittaker vector" if its restriction to $U_{0}$ contains a generic character.

If $\mu$ is a generic character of $U_{0}$ and $\pi$ a representation of $G$ such that $\left.\pi\right|_{U_{0}}$ contains $\mu$, we say that $\pi$ has a $\mu$-Whittaker vector.

Let $\psi$ be a non-trivial additive character of $k$. Then any one-dimensional character $\mu$ of $U_{0}$ may be represented in the form $I+x \mapsto \psi\left(\sum_{i=1}^{n-1} a_{i} x_{i, i+1}\right)=\mu(I+x)$ for some choice of $\left(a_{1}, \cdots, a_{n-1}\right) \in k^{n-1}$ ( $x$ an upper triangular nilpotent matrix). Clearly, $\mu$ is generic if and only if $\prod_{i=1}^{n-1} a_{i} \neq 0$. The diagonal group $T_{0}$ normalizes $U_{0}$ and acts on the set of one-dimensional characters of $U_{0}$. With respect to this action the set of generic characters comprise a single orbit. From this we obtain:

Lemma 5.1. Let $\pi$ be an irreducible representation of $G$. Let $\mu, \mu^{\prime}$ be generic characters of $U_{0}$. Then the multiplicities of $\mu$ and $\mu^{\prime}$ in $\left.\pi\right|_{U_{0}}$ are equal.

Let $X_{k}(1 \leq k<n)$ denote the subgroup of $U_{0}$ which consists of all matrices $I+x$ ( $x$ upper triangular nilpotent) such that the first $n-k$ columns of $x$ have only zero entries.

There is a folk argument, probably due to Gelfand/Graev or Gelfand/Kazhdan GK, to prove that any cuspidal representation of $G$ has a Whittaker vector. Here is this argument:

Lemma 5.2. Let $\pi$ be a cuspidal representation of $G$. Then $\pi$ is generic.

Proof. Let $(\pi, V)$ be an irreducible cuspidal representation of $G$ and let $\mathcal{P}=$ $\left(G_{n-1} \times G_{1}\right) \ltimes X_{1}$ be the standard "large" parabolic subgroup of $G$. Since $\pi$ is cuspidal, $\left.\pi\right|_{X_{1}}$ cannot contain $\mathbf{1}_{X_{1}}$. Therefore, since $X_{1}$ is isomorphic to the abelian group (under addition) $k^{n-1},\left.\pi\right|_{X_{1}}$ is a direct sum of non-trivial additive characters. Since $X_{1}-\{I\}$ is a single orbit under $G_{n-1} \times G_{1}$ acting by conjugation on $X_{1}$, every non-trivial (one-dimensional) character of $X_{1}$ occurs in $\left.\pi\right|_{X_{1}}$. In particular, the character $\mu_{1}:\left(x_{1, n}, \cdots, x_{n-1, n}\right) \mapsto \psi\left(x_{n-1, n}\right)$ occurs in $\left.\pi\right|_{X_{1}}$. Thus, we have proved the existence of a subspace $0 \neq V_{1} \subset V$ which transforms under $X_{1}$ as the isotypy component corresponding to $\mu_{1}$. We argue by induction to prove that for $1 \leq k<n$ there exists a non-zero isotypy subspace $V_{k} \subset V$ for $\left.\pi\right|_{X_{k}}$ which transforms as the character $\mu_{k}: I+x \mapsto \psi\left(x_{n-k, n-k+1}+\cdots+x_{n-1, n}\right)$. We have verified our claim for $k=1$ and assume it for $1 \leq k<n-1$. Let $U$ be the unipotent radical of the maximal parabolic subgroup $\left(G_{n-k-1} \times G_{k+1}\right) \ltimes U$. Since $\pi$ is cuspidal and since $\left.\pi\right|_{U \cap X_{k}}$ acts trivially on $V_{k}$, it follows that $\left.\pi\right|_{\left(G_{n-k} \times\{1, \cdots, 1\}\right) \cap U}$ is a direct sum of non-trivial characters on $V_{k}$. Since $G_{n-k-1} \times\{1, \cdots, 1\}$ stabilizes $V_{k}$ and acts transitively on $U \cap\left(G_{n-k} \times\{1, \cdots, 1\}-\{I\}\right)$, there exists a subspace $0 \neq V_{k+1} \subset V_{k}$ such that on $V_{k+1}$ we have a multiple of the character $I+x \mapsto \psi\left(x_{n-k-1, n-k}\right)$ for $I+x \in U \cap G_{n-k} \times\{1, \cdots, 1\}=X_{k+1} \cap G_{n-k} \times\{1, \cdots, 1\}$. Thus, on $V_{k+1}$ we also have a multiple of the character $\mu_{k+1}: I+x \mapsto \psi\left(x_{n-k-1, n-k}+\cdots+x_{n-1, n}\right)$ of the group $X_{k+1}$. By induction it follows that there is a non-zero subspace $V_{n-1}$ 
on which the generic character $I+x \mapsto \psi\left(x_{1,2}+\cdots+x_{n-1, n}\right)$ of $X_{n-1}=U_{0}$ acts. This proves that $\pi$ cuspidal implies that $\pi$ is generic.

Let us recall the following assertion of S. I. Gelfand. Gelfand gives a short proof of this result in GE2], based on the theorem of Gelfand/Graev (see Corollary 5.4 below). We shall derive Gelfand/Graev's result as a consequence of our development, so we give a different proof for S. I. Gelfand's assertion.

Lemma 5.3 (S. I. Gelfand [GE1], GE2]). Let $\mu$ be a generic character of $U_{0}$ and let $\chi$ be a character of the center $Z \cong k^{\times}$of $G$. Let $\mathcal{P}=\left(G_{n-1} \times G_{1}\right) \ltimes X_{1}$ denote the standard large parabolic subgroup of $G$. Then the representation $\rho(\chi)=$ Ind $_{Z \times U_{0}}^{\mathcal{P}} \chi \otimes \mu$ is irreducible and independent of $\mu$. Moreover, for any irreducible cuspidal representation $\pi$ of $G$ with central character $\chi,\left.\rho(\chi) \sim \pi\right|_{\mathcal{P}}$.

Proof. Clearly, $\operatorname{Ind}_{Z U_{0}}^{T_{0} U_{0}}(\chi \otimes \mu)$ is a representation of degree $\left[T_{0} U_{0}: Z U_{0}\right]=(q-1)^{n-1}$ with the property that its restriction to $U_{0}$ consists of the direct sum of all the characters of $U_{0}$ which are conjugate to $\mu$ under the action of $T_{0}$. Thus every generic character of $U_{0}$ occurs in this representation and each occurs simply. By the transitivity of induction this proves that $\rho(\chi)$ does not depend upon $\mu$ generic. For the irreducibility we argue by induction, the case $n=1$ being obvious. Assume that for $k<n$ the representation $\rho(\chi)$ is irreducible and that $\left.\rho(\chi) \sim \pi\right|_{\mathcal{P}}$. Let $\left(\pi_{1} \otimes \lambda, W\right)$ be the tensor product of an irreducible cuspidal representation of $G_{n-1}$ and a character of $G_{1}$ such that $\left.\pi_{1} \otimes \lambda\right|_{Z}$ is the scalar representation $\chi \cdot I_{\pi_{1} \otimes \lambda}$. Consider $J=\operatorname{Ind}_{G_{n-1} \times G_{1}}^{\mathcal{P}}\left(\pi_{1} \otimes \lambda\right)$. The commuting algebra of $J$ is the convolution algebra of $\operatorname{End}(W)$-valued functions $h$ which satisfy $h\left(g p g^{\prime}\right)=\left(\pi_{1} \otimes \lambda\right)(g) h(p)\left(\pi_{1} \otimes \lambda\right)\left(g^{\prime}\right)$ for all $g, g^{\prime} \in G_{n-1} \times G_{1}, p \in \mathcal{P}$. Since the non-zero elements of $k^{n-1}$ comprise a single orbit under the action of $G_{n-1}$, it is obvious that there are exactly two $G_{n-1} \times G_{1} \backslash \mathcal{P} / G_{n-1} \times G_{1}$ double cosets, one with representative the identity element of $X_{1}$ and the other with representative any non-identity element of $X_{1}$. We claim that the commuting algebra of $J$ is two-dimensional. Clearly, the double coset with representative the identity element of $X_{1}$ supports a one-dimensional subspace. Let us show that the other double coset also supports only a one-dimensional subspace. To see this note that any function with support in this double coset must satisfy

$$
\left(\pi_{1} \otimes \lambda\right)(g) h(x)=h(g x)=h(x g)=h(x)\left(\pi_{1} \otimes \lambda\right)(g)
$$

at $I \neq x \in X_{1}$ whenever $g x g^{-1}=x$ with $g \in G_{n-1} \times G_{1}$. We observe that the set of $g \in G_{n-1} \times G_{1}$ such that $g x g^{-1}=x$ for a non-identity element $x \in X_{1}$ is a group of the form $\mathcal{P}^{\prime} \times G_{1}$, where $\mathcal{P}^{\prime}$ is a large parabolic subgroup of $G_{n-1}$. By induction $\left.\pi_{1}\right|_{\mathcal{P}^{\prime}}$ is irreducible; therefore, by Schur's Lemma, the space of functions satisfying $(*)$ is one-dimensional. Thus the commuting algebra of $J$ is two-dimensional and therefore $J$ has two inequivalent irreducible component representations. Now $J$ obviously contains the "lift" to $\mathcal{P}$ of $\pi_{1} \otimes \lambda$ as a subrepresentation. The dimension of $J$ is

$$
\operatorname{dim}\left(\pi_{1}\right) q^{n-1}=q^{n-1} \prod_{j=1}^{n-2}\left(q^{j}-1\right),
$$

and the dimension of the complement of the lift is

$$
\prod_{j=1}^{n-1}\left(q^{j}-1\right)=\left[\mathcal{P}: Z U_{0}\right]=\operatorname{dim}(\rho(\chi)) .
$$


Clearly, the complement of the lift contains a Whittaker vector, from which we conclude via Frobenius reciprocity and by matching dimensions that it is equivalent to $\rho(\chi)$ and that $\rho(\chi)$ is irreducible. By Lemma 5.2 any irreducible cuspidal representation $\pi$ is generic, so, by Frobenius reciprocity, if the central character of $\pi$ is $\chi,\left.\pi\right|_{\mathcal{P}}$ contains a subrepresentation which is equivalent to the irreducible representation $\rho(\chi)$. By Green's results,

$$
\operatorname{dim}(\pi)=\prod_{j=1}^{n-1}\left(q^{j}-1\right),
$$

so $\left.\pi\right|_{\mathcal{P}} \sim \rho(\chi)$.

Corollary 5.4. Let $\pi$ be an irreducible cuspidal representation of $G$. Then for any generic character $\mu$ of $U_{0}$ the multiplicity of $\mu$ in $\left.\pi\right|_{U_{0}}$ is exactly one.

Proof. This is now immediate from the irreducibility of the induced representation $\rho(\chi)$ combined with Frobenius reciprocity.

We extend the definition of Whittaker vector to apply to any irreducible representation $\pi=\pi_{1} \otimes \cdots \otimes \pi_{r}$ of $G_{m_{1}} \times \cdots \times G_{m_{r}}$ by saying that $\pi$ has a Whittaker vector if each of the factors $\pi_{i}$ has one. Again if $\pi$ has a $\mu$-Whittaker vector with respect to one generic character $\mu$ of $U_{0} \cap\left(G_{m_{1}} \times \cdots \times G_{m_{r}}\right)$, then it has a Whittaker vector with respect to every other such character. The multiplicity for each generic character is clearly the same. We shall say that $\pi$ has a $\mu$-Whittaker vector if we want to specify a particular character $\mu$ of $U_{0} \cap\left(G_{m_{1}} \times \cdots \times G_{m_{r}}\right)$.

Theorem 5.5 (cf. Rodier $[\mathrm{ROD}]$ ). Let $L=G_{m_{1}} \times \cdots \times G_{m_{r}}\left(m_{1}+\cdots+m_{r}=n\right)$ be a block diagonal subgroup of $G$. Let $P=L \ltimes U$ be the parabolic subgroup of $G$ which contains $P_{0}$ and has $L$ as Levi factor. Let $\pi=\pi_{1} \otimes \cdots \otimes \pi_{r}$ be any representation of $L$, irreducible or not, which has, up to scalar factors, exactly one $\mu_{0}$-Whittaker vector, where $\mu_{0}$ is a generic character of $U_{0} \cap L$. Then $\Pi=\operatorname{Ind}_{P}^{G} \pi$ has, up to scalar factors, exactly one $\mu$-Whittaker vector for any generic character $\mu$ of $U_{0}$.

Proof. To avoid some minor technicalities we replace $U_{0}$ by its opposite $\bar{U}_{0}$; we show that there is, up to scalar factors, a unique $\mu$-Whittaker vector (i.e. for $\bar{U}_{0}$ ) with $\mu$ of $\bar{U}_{0}$ such that $\left.\mu\right|_{\bar{U}_{0} \cap L}=\left.\mu_{0}\right|_{\bar{U}_{0} \cap L}$. It is enough to prove the theorem for $\bar{U}_{0}$, because $U_{0}$ and $\bar{U}_{0}$ are conjugate. To prove existence we consider the subset $P \bar{U} \cong P \times \bar{U}$ of $G$, where $\bar{U}$ denotes the opposite of $U$. Let $\mu_{1}=\left.\mu\right|_{\bar{U}}$. Then for any $\bar{u}_{0} \in \bar{U}_{0}$ we have $\bar{u}_{0}=\bar{u}_{L} \bar{u}$ with $\bar{u}_{L} \in\left(\bar{U}_{0} \cap L\right)$ and $\bar{u} \in \bar{U}$. Let $v=v_{\mu_{0}}$ be the, up to scalar factors, unique vector in the representation space $V_{\pi}$ of $\pi$ which transforms as $\mu_{0}$ under $\bar{U}_{0} \cap L$. Let $h: G \rightarrow V_{\pi}$ be defined such that $h(p \bar{u})=\mu_{1}(\bar{u}) \pi(p) v_{\mu_{0}}$ for any $p \in P$ and $\bar{u} \in \bar{U}$. Assume that the support of $h$ lies in $P \bar{U}$. Then clearly $h$ is a $\mu$-Whittaker vector for $\Pi$; it is also clear that $h$ is, up to scalar factors, the unique $\mu$-Whittaker vector for $\Pi$ with support in $P \bar{U}$. We must show that there is no $\mu$-Whittaker vector for $\Pi$ which has support in a coset of the form $P w \bar{U}$, where $w$ is a permutation matrix not lying in $L$. By transitivity of parabolic induction and arguing by induction on the parabolic rank of $P$ it is sufficient to consider the p-rank one case, $r=2$. Using the fact that the p-rank is one, let $\lambda$ be the weight which is one on the positive root occurring in the Lie algebra of $U$ and is orthogonal to all the other simple roots, i.e. to the set of simple roots which occur in the Lie algebra of $L \cap U_{0}$. If $w$ fixes $\lambda$, then $w$ belongs to the group generated by the set of 
reflections in the group of permutation matrices which fix $\lambda$ [BOU, p. 75, Prop.1]. Since $\lambda$ lies in the closure of a positive chamber, this group is generated by the set of reflections which correspond to the set of simple roots which are orthogonal to $\lambda$, precisely to the set of simple roots which occur in the Lie algebra of $L \cap U_{0}$. This reflection group is just the set of all permutation matrices lying in $L$. If $w$ represents a double coset $P w \bar{U} \neq P \bar{U}$, then $w \notin L$ and therefore $w$ does not fix $\lambda$. Since

$$
\left(\lambda, w^{-1} \alpha\right)=(w \lambda, \alpha)<0
$$

for some simple root, it follows that some subdiagonal one-parameter subgroup of $\bar{U}_{0}$ is conjugated by $w$ into $U$. Therefore, for any $h: P w \bar{U} \rightarrow V_{\pi}$ which satisfies $h(l u w \bar{u})=\pi(l) h(w \bar{u})$ for $l \in L, u \in U, \bar{u} \in \bar{U}$, we have

$$
\Pi\left(\bar{u}_{0}\right) h(w)=h\left(w \bar{u}_{0}\right)=h\left(w \bar{u}_{0} w^{-1} \cdot w\right) .
$$

In this case it follows that $\Pi\left(\bar{u}_{0}\right) h(w)=h(w)$. This implies that $h$ is not an eigenfunction for $\left.\Pi\right|_{\bar{U}_{0}}$ which transforms according to a generic character of $\bar{U}_{0}$. We conclude that, up to scalar factors, there is only one $\mu$-Whittaker vector in the representation space of $\Pi$.

Corollary 5.6 (Gelfand/Graev [GG]). Let $\pi$ be an irreducible representation of $G$. For any generic character $\mu$ of $U_{0}$ the representation $\pi$ has at most a onedimensional space of $\mu$-Whittaker vectors.

Proof. Since every irreducible representation of $G$ occurs as a subrepresentation of $\operatorname{Ind}_{P}^{G} \sigma$ with $\sigma$ a cuspidal representation of a Levi factor $L$ lifted to $P=L \ltimes U$, our claim is an immediate consequence of Corollary 5.4 and Theorem 5.5.

Corollary 5.7. Let $\sigma$ be a cuspidal representation of $G_{s}$, where $n=s v$. Let $L=$ $\left[G_{s}\right]^{v}$ be a block-diagonal subgroup of $G$ and let $P=L \ltimes U$ be a parabolic subgroup of $G$ with $L$ as Levi factor. The only component of $\Pi=\operatorname{Ind}_{P}^{G} \sigma^{\otimes v}$ with a Whittaker vector is the GS component.

Proof. By Corollary 5.4 and Theorem 5.5, $\Pi$ contains exactly one $\mu$-Whittaker vector, up to scalar multiples, for any generic character $\mu$ of $U_{0}$. Therefore, exactly one component of $\Pi$ can have Whittaker vectors. This component $\pi$ of $\Pi$ must occur simply, since the property of having a Whittaker vector is clearly invariant under equivalence. Thus the only possible components of $\Pi$ which can have Whittaker vectors are the GT and GS components. However, if a component $\pi$ of $\Pi$ has a Whittaker vector, then $\left.\pi\right|_{\mathcal{P}}$ must contain an irreducible component of dimension $\left[\mathcal{P}: Z U_{0}\right]=\prod_{j=1}^{n-1}\left(q^{j}-1\right)$. Thus, the dimension of $\pi$ must be at least as large as this dimension. By $\S 3,(3)$, the dimension of the GT representation is too small; note also that $\S 3,(5)$ implies that the dimension of the GS representation is large enough to be consistent with its having Whittaker vectors. Since one and only one component of $\Pi$ is generic, it is the GS component which is generic.

\section{GS Characters on the Regular Elliptic Set}

Our main result characterizes and parameterizes the set of cuspidal and generalized Steinberg representations by their character values on the regular elliptic set of $G$. 
Theorem 6.1. Let $\chi_{s}=\theta^{k}$ be a regular character of $k_{s}^{\times}$and let $\sigma=\sigma_{\chi_{s}}$ be the cuspidal representation of $G_{s}$ with character $U_{\chi_{s}}(\gamma)=(-1)^{s-1} S\left(\chi_{s}\right)(\gamma)$ for any regular elliptic element $\gamma \in G_{s}$. Consider $\sigma^{\otimes v}$ as a representation of a parabolic subgroup $P=L \ltimes U$ with the Levi factor $L$ of $P$ isomorphic to $G_{s} \times \cdots \times G_{s}$. Let $\Sigma$ be the generalized Steinberg component of the induced representation $I\left(P, \sigma^{\otimes v}\right)$. Then the character $U=U_{\Sigma}$ is represented on the regular elliptic set of $G$ by the formula

$$
\begin{aligned}
U(\xi) & =(-1)^{n-1} \sum_{j=0}^{s-1} \mathrm{~N}_{k_{n} \mid k_{s}}^{*}\left(\chi_{s}\right)\left(\xi^{q^{j}}\right) \\
& =(-1)^{n-1} \sum_{j=0}^{s-1} \mathrm{~N}_{k_{n} \mid k_{s}}^{*}\left(\chi_{s}\right)^{q^{j}}(\xi) \\
& =(-1)^{n-1} S\left(\chi_{n}\right)(\xi),
\end{aligned}
$$

where we identify any regular elliptic element $\xi \in G$ with a primitive element in $k_{n}^{\times}$ and

$$
\mathrm{N}_{k_{n} \mid k_{s}}^{*}\left(\chi_{s}\right)=\chi_{s} \circ \mathrm{N}_{k_{n} \mid k_{s}}=\chi_{n} \in X_{n} .
$$

Proof. Green [GR, p.439, Theorem 12] represents the character $U=(-1)^{n-v} I_{s}^{k}\left[1^{v}\right]$ in the form

$$
U_{\rho}\left(\xi^{\rho}\right)=(-1)^{n-v} \chi_{\pi}^{1^{v}} \prod_{\ell} \prod_{i=1}^{p_{\ell}} T_{s, \ell}\left(k: \xi_{s \ell, i}\right)
$$

for any conjugacy class which corresponds to a characteristic polynomial with distinct roots (a "principal conjugacy class"). In (9) $\pi$ is a partition of $v, \chi_{\pi}^{1^{v}}$ is the sign character of $\mathfrak{S}_{v}$ evaluated at $\pi$, and $\rho$ is the partition $s \pi$ of $n$. These partitions depend upon the factorization of the minimal (or characteristic) polynomial of the conjugacy class. If the argument is regular elliptic, we have $\pi=\left(v^{1}\right)$ and $\rho=s \pi=\left(n^{1}\right)$, which implies that $\chi_{\pi}^{1^{v}}=\chi_{v}^{1^{v}}=(-1)^{v-1}$. Since $\pi=\left(v^{1}\right)$, the product $\Pi_{\ell}$ consists of one factor corresponding to $\ell=v$, and $p_{\ell}=p_{v}=1$. Therefore, since

$$
(-1)^{n-v} \chi_{v}^{1^{v}}=(-1)^{n-v}(-1)^{v-1}=(-1)^{n-1}
$$

and since the product in (9) collapses to a single factor corresponding to $p_{v}=1$, we have

$$
U_{\rho}\left(\xi^{\rho}\right)=U(\xi)=(-1)^{n-1} T_{s, v}\left(k: \xi_{n, 1}\right) .
$$

In [GR, p. 417, Definition 3.1] we find that

$$
T_{s, v}(k: \xi)=\left(\theta^{k} \circ \mathrm{N}_{k_{n} \mid k_{s}}\right)(\xi)+\left(\theta^{k} \circ \mathrm{N}_{k_{n} \mid k_{s}}\right)^{q}(\xi)+\cdots+\left(\theta^{k} \circ \mathrm{N}_{k_{n} \mid k_{s}}\right)^{q^{s-1}}(\xi),
$$

where $\xi=\xi_{n, 1}$ is a root of the characteristic (or minimal) polynomial defining the conjugacy class, i.e. a regular elliptic element of $G$. Hence (9) implies (8).

Corollary 6.2. For every $n \geq 1$ there is a bijective correspondence $\left[\chi_{n}\right] \mapsto \Sigma_{\chi_{n}}$ between the parameter set $X_{n} /\langle\phi\rangle$ of Galois orbits of characters of $k_{n}^{\times}$and the set of cuspidal and generalized Steinberg representations of $G_{n}$. If $\left[\chi_{n}\right] \subset X_{n}$ consists of regular characters, then the representation $\Sigma_{\chi_{n}}$ of $G_{n}$ is a cuspidal representation. If $\left[\chi_{n}\right]=\left[N_{k_{n} \mid k_{s}}^{*} \chi_{s}\right]$, where $\chi_{s} \in X_{s}$ is regular, $s \mid n$, and $s<n$, then $\Sigma_{\chi_{n}}$ is the GS representation which occurs as a component of the induced representation $I\left(P, \sigma^{\otimes v}\right)$ 
$(s v=n)$, where $P=\left[G_{s}\right]^{v} \ltimes U$ is a parabolic subgroup of $G_{n}$ and $\sigma=\Sigma_{\chi_{s}}$ is the cuspidal representation of $G_{s}$ which corresponds to $\left[\chi_{s}\right]$. The inverse mapping is $\Sigma \mapsto\left[\chi_{n}\right]$, where the character of $\Sigma$ is $(-1)^{n-1} \sum_{\eta \in\left[\chi_{n}\right]} \eta$, on the regular elliptic set of $G_{n}$.

Proof. Theorem 1.1 implies that the orbit $\left[\chi_{n}\right]$ may be recovered from the restriction of the character of $\Sigma$ to the regular elliptic set of $G_{n}$.

Remark 6.3.

1. The set $\tilde{X} /\langle\phi\rangle$ corresponds bijectively to the set of cuspidal representations of all groups $G_{n}(n \geq 1)$. This correspondence is given as follows. Let $[\tilde{\chi}] \in \tilde{X} /\langle\phi\rangle$ and assume that $|[\tilde{\chi}]|=s \geq 1$. Then

$$
[\tilde{\chi}]=\bigcup_{m: s \mid m}\left[\chi_{m}\right] \subset \coprod_{m=1}^{\infty} X_{m}=X
$$

where $\left[\chi_{m}\right] \subset X_{m}$. Only $[\tilde{\chi}] \cap X_{s}=\left[\chi_{s}\right]$ consists of regular characters; to $\left[\chi_{s}\right]$ corresponds a cuspidal representation $\sigma_{\chi_{s}}$ of $G_{s}$.

2. Fix $\tilde{\chi} \in \tilde{X}$, assume that $|[\tilde{\chi}]|=s \geq 1$, and let $\sigma_{\chi_{s}}$ be the cuspidal representation of $G_{s}$ which corresponds to $[\tilde{\chi}]$. For all $m$ such that $s \mid m(s<m)$ there is a unique GS representation $\Sigma$ of $G_{m}$ such that $\Sigma$ corresponds to the orbit $\left[\chi_{m}\right]=X_{m} \cap[\tilde{\chi}]$ and such that $\Sigma$ has $\sigma_{\chi_{s}}$ as its cuspidal base representation. The mapping $\left[\chi_{m}\right] \mapsto \Sigma=\Sigma_{\chi_{m}}$ was described in Corollary 6.2.

3. Let $\Sigma$ be the cuspidal or GS representation of $G_{m}(s \mid m, s \leq m)$ which corresponds to $[\tilde{\chi}] \cap X_{m}$. The function

$$
(-1)^{m-1} S(\tilde{\chi})_{m}: \mathcal{R}_{m} \rightarrow \mathbb{C},
$$

$S(\tilde{\chi})_{m}$ as in $\S 1,\left(1^{\prime}\right)$, represents the character of $\Sigma$ on the regular elliptic set of $G_{m}$. The orbit $[\tilde{\chi}]$ can be recovered from this function (for any $m$ ).

4. The set $X /\langle\phi\rangle$ is in bijective correspondence with the set of cuspidal and GS representations of all the groups $G_{m}(m \geq 1)$. The subset $[\tilde{\chi}] \subset X /\langle\phi\rangle$ parameterizes the set of all cuspidal and GS representations which have $\sigma_{\chi_{s}}$ as cuspidal base representation.

We represent the above remark by the following commutative diagram in which $\mathbb{N}$ denotes the set of positive integers:

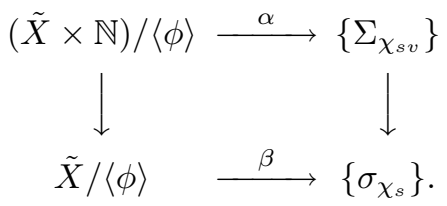

In the above diagram, assuming that $|[\tilde{\chi}]|=s, \alpha([\tilde{\chi}], v)=\Sigma_{\chi_{s v}}$, where $\Sigma_{\chi_{s v}}$ is the GS representation of $G_{s v}$ with $\sigma_{\chi_{s}}$ as cuspidal base representation. We also have $\beta([\tilde{\chi}])=\alpha([\tilde{\chi}], 1)=\sigma_{\chi_{s}}$, a cuspidal representation of $G_{s}$. The left vertical arrow "forgets" the integer component and the right vertical arrow associates to a GS representation its cuspidal base representation. Of course $\chi_{s v} \sim \chi_{s}$ for all $v \in \mathbb{N}$.

\section{REFERENCES}

[ART] E. Artin, The Orders of the Linear Groups, The Collected Papers of Emil Artin, AddisonWesley, 1965, pp. 355-397. MR 31:1159 
[BZ] I. N. Bernshtein and A. V. Zelevinskii, Representations of the Group $G L(n, F)$ Where $F$ is a Non-Archimedean Local Field, Russian Math. Surveys 31:3 (1976), 1-68. [MR 54:12988

[BV] G. D. Birkhoff and H. S. Vandiver, On the Integral Divisors of $a^{n}-b^{n}$, Ann. of Math. 5 (1904), 173-180.

[BOU] N. Bourbaki, Groupes et algèbres de Lie, Chapitres 4, 5, et 6, Éléments de mathématique, Fascicule XXXIV, Hermann, 1968. MR 39:1590

[CAR] R. W. Carter, Finite Groups of Lie Type: Conjugacy Classes and Complex Characters, A Wiley-Interscience Publication, Wiley Classics Library Edition Published 1993, John Wiley \& Sons, 1985. MR 94k:20020

[CUR] C. W. Curtis, Truncation and Duality in the Character Ring of a Finite Group of Lie Type, Journal of Algebra 62 (1980), 320-332. [MR 81e:20011]

[DL] P. Deligne and G. Lusztig, Representations of Reductive Groups Over Finite Fields, Ann. of Math. 103 (1976), 103-161. MR 52:14076

[GG] I. M. Gelfand and M. I. Graev, Soviet Math. Dokl. 3 (1962), 1646.

[GK] I. M. Gelfand and D. Kazhdan, Representations of the Group GL $(n, K)$ Where $K$ is a Local Field, Lie Groups and Their Representations, A Halsted Press Book, John Wiley \& Sons, 1971, pp. 95-118. MR 53:8334

[GE1] S. I. Gelfand, Representations of the Full Linear Group over a Finite Field, Mat. Sb. 83(N. S. 125) (1970), 15-41. MR 42:7797

[GE2] S. I. Gelfand, Representations of the General Linear Group Over a Finite Field, Lie Groups and Their Representations, A Halsted Press Book, John Wiley \& Sons, 1971, pp. 119-132. MR 56:490

[GR] J. A. Green, The Characters of the Finite General Linear Groups, Trans. Amer. Math. Soc. 80 (1955), 402-447. MR 17:345e

$[\mathrm{HM}]$ R. E. Howe, with the collaboration of A. Moy, Harish-Chandra Homomorphisms for $\mathfrak{p}-$ adic Groups, Expository Lectures from the CBMS Conference held at the University of Chicago August 8-12, 1983, Amer. Math. Soc., 1985. MR 87h:22023

[HL1] R. B. Howlett and G. I. Lehrer, Induced Cuspidal Representations and Generalized Hecke Rings, Invent. Math. 58 (1980), 37-64. MR 81j:20017

[HL2] _ A Comparison Theorem and Other Formulae in the Character Ring of a Finite Group of Lie Type, Contemp. Math., vol. 9, Amer. Math. Soc., 1981, pp. 285-288. MR 84b:20055

[IW] N. Iwahori, Generalized Tits System (Bruhat Decomposition) on $\mathfrak{p}$-adic Semisimple Groups, Algebraic Groups and Discontinuous Subgroups, Proc. Sympos. in Math., vol. IX, 1966, pp. 71-83. MR 35:6693

[IM] N. Iwahori and H. Matsumoto, On Some Bruhat Decomposition and the Structure of the Hecke Rings of the $\mathfrak{p}$-adic Chevalley Groups, Publ. Math., I. H. E. S. 25 (1965), 5-48. MR 32:2486

[L] G. Lusztig, Characters of Reductive Groups over a Finite Field, Ann. Math. Stud., vol. 107, Princeton Univ. Press, 1984. MR 86j:20038

[MAC] I. G. Macdonald, Zeta Functions Attached to Finite General Linear Groups, Math. Ann. 249 (1980), 1-15. MR 81m:20062

[ROD] F. Rodier, Whittaker Models for Admissible Representations of Reductive $\mathfrak{p}$-adic Split Groups, Harmonic Analysis on Homogeneous Spaces, Proc. of Sympos. in Pure Math., vol. XXVI, Amer. Math. Soc., 1973, pp. 425-430. MR 50:7419

[SZ1] A. J. Silberger and E.-W. Zink, The Formal Degree of Discrete Series Representations of Central Simple Algebras Over p-Adic Fields, Max-Planck-Institut Für Mathematik.

[SZ2] A. J. Silberger and E.-W. Zink, Weak Explicit Matching of the Level Zero Discrete Series for Unit Groups of p-adic Simple Algebras, In preparation.

[ZS] K. Zsigmondy, Zur Theorie der Potenzreste, Monatsh. Math. Phys. 3 (1892), 265-284.

Department of Mathematics, Cleveland State University, Cleveland, Ohio 44115

E-mail address: silberger@math.csuohio.edu

Humboldt-Universität, FB Reine Mathematik, Unter den Linden 6, 10099 Berlin, GerMANY

E-mail address: zink@mathematik.hu-berlin.de 